\title{
Ant Lion Optimizer for Suppression of Ambipolar Conduction in Schottky Barrier Carbon Nanotube Field Effect Transistors
}

\section{Gagnesh Kumar ( $\nabla$ gagnesh78@gmail.com )}

NIT Hamirpur: National Institute of Technology Hamirpur

\section{Sunil Agrawal}

UIET: University Institute of Engineering and Technology

\section{Research Article}

Keywords: Ambipolar Conduction, Carbon nanotubes, high-k gate oxide, Schottky barrier

Posted Date: July 23rd, 2021

DOl: https://doi.org/10.21203/rs.3.rs-619648/v1

License: (c) (1) This work is licensed under a Creative Commons Attribution 4.0 International License.

Read Full License 


\title{
1 Ant Lion Optimizer for Suppression of Ambipolar
}

\section{Conduction in Schottky Barrier Carbon Nanotube Field}

\section{Effect Transistors}

\author{
4 Gagnesh Kumar $^{1 *}$ and Sunil Agrawal ${ }^{2}$ \\ * Correspondence: gagnesh85@gmail.com \\ 7 the date of receipt and acceptance should be inserted later
}

8 Abstract A mathematical model and experimental analysis of the effect of oxide 9 thickness on ambient conduction is provided in the Schottky Barrier Carbon Nan10 otubes (CNTs) Field Effect Transistor (SB-CNTFET). To develop them as the future 11 of IC (integrated circuit) technology, the suppression of ambipolar behaviour in SB12 CNTFET is imperative. The ambipolar nature of SB-CNTFET contributes to a high 13 amount of leakage current. $t o x \approx 49.91 \mathrm{~mm}$ uses a dielectric of gate oxide with a 14 thickness to inhibit the ambipolar behaviour. In an SB-CNTFET, the conductance 15 is regulated by the electrical field at the source/drain contacts and the band bend16 ing length at the contacts is determined by $t_{o x}$. Therefore, the prime parameter $t_{o x}$ 17 that affects the Schottky barrier width and the subthreshold area. The suppression of 18 ambipolar property is presented through experimental analysis. The SB-CNTFET is 19 produced using high-k dielectrics such as Zirconium dioxide. This work discusses the ${ }_{20}$ suppression of ambipolar activity in SB-CNTFETs without reducing the $I_{o n}$ current 21 using an appropriate dielectric with optimum thickness.

\footnotetext{
${ }^{1}$ Department of Electronics \& Communication, National Institute of Technology Hamirpur.

${ }^{2}$ Department of Electronics, UIET, Panjab University, Chandigarh.
} 


\section{Introduction}

The emerging trend towards the Internet of Things (IoT) has increased the demand of scaled-down low power devices with high-performance [1], [31], [3], [7], [10]. This need is addressed by the growth of nano-electronic devices based on novel materials such as semiconducting carbon nanotubes (CNTs) [36] with their outstanding nanoscale carrier mobility [8]. Owing to their ballistic electron transport, they can carry strong currents without scattering [14].

Among all novel nano electronic devices, the carbon nanotube field effect transistors (CNTFETs) are in the limelight for their promising device characteristics [4]. The CNTFETs also exhibit high frequency characteristics up to $10 \mathrm{GHz}$ [14]. In the recent applications, the CNTFETs are found in the gas sensing based on IoT, antennas based CNTs, and interconnect in the RF unit [5], [6], [30], [9], [11], [13]. CNTs can be used as ultrasensitive gas sensors for the next generation applications [32], [35].

There are some barriers, despite all these credentials, that restrict the commercial use of CNTFETs. One such barrier is discussed in this paper, i.e., the ambipolar behaviour of CNTFET that increases the OFF-state leakage current. The OFF current found at Schottky Barrier (SB) is due to the tunnelling of charge carrier. A metalcontacted CNTFET is favoured over a heavily doped semiconductor-contacted CNTFET because the metals substantially show lower parasitic resistance [18]. However, the doping techniques of CNTFET have not achieved any substantial maturity.

The focus of this work is on the Schottky Barrier CNTFET (SB-CNTFET) with metal interface. Previously, the only way to make the system unipolar was to dope the contact points extensively [18], [17]. Other effective ways to suppress SB-CNTFET's ambipolar behaviour are discussed: Channel halo doping in CMOS technologies is one of the techniques for reducing short-channel effects [16]. This technique is used to inhibit ambipolar activity in CNTFETs. There are p-type dopants added for some unique duration in the CNT channel. Since the source side of the channel is p-doped, all variations of the drain potential are screened. It has been shown that the halo doping technique lowers the leakage current. Naderi et al. launched another new unit, a Linear Doped Channel (LDC) CNTFET [27], [29]. The halo doping is linearly distributed across the channel rather than doping on one end of the channel, with a maximum concentration decreasing linearly to zero on the source side as it goes to the 
drain side of the channel. The gate structure engineering can also achieve the suppression of CNTFETs' ambipolar behaviour. The ambipolar behaviour [34] is suppressed by a vertical partial gate where the gate only covers a portion of the channel. The absence of a gate on the drain side makes the SB on this side thick, ensuring unipolar behaviour. The same impact of the gate without controlling the channel may also be generated by a deep trench inserted under the CNT near the source or drain contact. The back gate under CNT does not control the trench area [22], [23]. The absence of control over that portion of the channel makes the SB thick on the side of the source/drain, thus suppressing ambipolar behaviour. All of these approaches focus on advanced fabrication techniques. The study of nanotube diameter function and the effect of gate oxide thickness found that the thin gate oxide $(4 \mathrm{~nm})$ CNTFETs exhibited ambipolar characteristics [14], [15]. The gate oxide thickness approximately equals to the thickness of the SB [33]. Because of the one dimensional structure of the CNTs, the gate capacitance, $C_{o x}$, is inversely proportional to the logarithm of the oxide thickness, $t_{o x}$, in long channel devices. The tunnelling in the barrier and ambipolar conduction is achieved due to the scaling of $t_{o x}$ aggressively. The charge in the silicon MOSFET channel is affected by $t_{o x}$ through gate capacitance which affects the conductance in the $\mathrm{ON}$ state. It is the electric field at the source/drain contacts that regulate the conductance in an SB CNTFET, and $t_{o x}$ defines the band bending length at the contacts. The $t_{o x}$ is, therefore, the primary parameter which affects subthreshold region current and Schottky barrier width. The fact that CNTFETs with thin gate oxide appear to be ambipolar with almost symmetrical features is exposed by experimental results [37], [2]. A backgated SB CNTFET exhibited ambipolar characteristics with $2 \mathrm{~nm}$ and $5 \mathrm{~nm}$ thick gate oxide. An asymmetric barrier height led to asymmetric electron and hole conduction when the gate insulator was thick $(40 \mathrm{~nm})$ [38]. Thus, by providing a thick dielectric gate, ambipolar behaviour is suppressed. To suppress ambipolar conduction, it is, therefore, necessary to obtain the optimal values of gate oxide thickness.

The dense dielectric gate, however, decreases the current due to tunnelling. A high $-\mathrm{K}$ dielectric such as Zirconium dioxide is used to compensate for this decrease in current. High-K dielectrics are commonly used in scaled-down devices with thin gate oxides to reduce the leakage current. The purpose of a high-K dielectric is to increase conductance through thermionic emission. The on-current is, therefore, not 
affected. An SB-CNTFET with $50 \mathrm{~nm}$ channel length was manufactured and characterised to demonstrate the occurrence of ambipolar conduction in SB CNTFETs with CNTs as a channel and thin gate oxide. The findings indicate that when compared to the on-currents recorded in the literature, where low- $\mathrm{K}$ dielectric such as $\mathrm{SiO}_{2}$ is used, the high $-\mathrm{K}$ dielectric ensures a better on current [19], [28], [25], [26]. The rise in thickness, toxicity, guarantees that ambipolar activity is suppressed.

This paper is structured as follows: the mathematical model of the proposed SB CNTFET and the optimization using particle swarm intelligence are discussed in Section 2. The multiobjective optimization is presented in Section 3 . Section 4 addresses the findings and inferences. The paper ends with Section 5 .

\section{Modelling the SB CNTFET}

As the channel exhibits ambipolar activity, an SB CNTFET with a single-walled CNT. The model of the SB CNTFET has been shown in Fig. 1 . The distribution functions in Eq. (1) and Eq. (2) are given by considering the source and the drain terminals Schottky barriers.

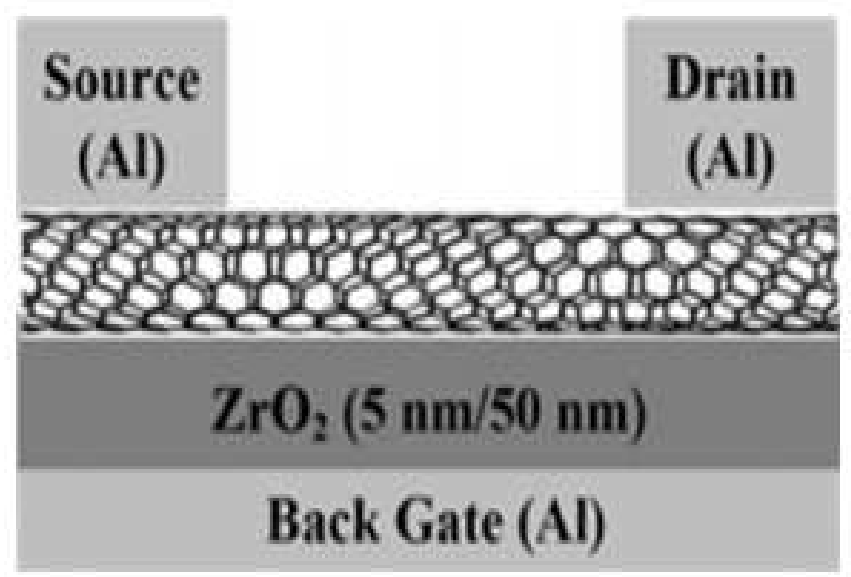

Fig. 1: Cross-sectional view of SB-CNTFET 


$$
f^{+}=\frac{T_{s} f_{s}+T_{d} f_{d}-T_{s} T_{d} f_{d}}{1-\left(T_{s}-1\right) \cdot\left(T_{d}-1\right)}
$$

104

$$
f^{-}=\frac{T_{d} f_{d}+T_{s} f_{s}-T_{s} T_{d} f_{s}}{1-\left(T_{s}-1\right) \cdot\left(T_{d}-1\right)}
$$

Applying the formula of Landauer-Büttiker the ballistic current $I_{d}$, is determined in Eq. (3).

$$
I_{d}=\frac{2 q}{h} \Sigma \int_{E_{\text {imin }}}^{E_{\text {imax }}}\left(f^{+}(E, \mu)-f^{-}(E, \mu)\right) d E
$$

The method was adopted in John et al. [20] for the SB-CNTFETs' self-consistent simulations, solving the Schrödinger equation as in Eq. (4),

$$
-\frac{h^{2}}{2 m^{*}} \frac{\partial \varphi_{s}}{\partial x^{2}}-(U-\varepsilon) \varphi=0
$$

are entered into the Poisson's equation as in Eq. 97

$$
\Delta \varepsilon \Delta \phi=-\frac{q(p-n) \delta\left(\rho-\rho_{C N T}\right)}{2 \pi \rho}
$$

The $\delta$, here, denotes the Dirac-delta function, which defines the charge density of CNT. Electrons and holes are known as sheets of charges. We assume that the charges 


\section{CNTFET.}

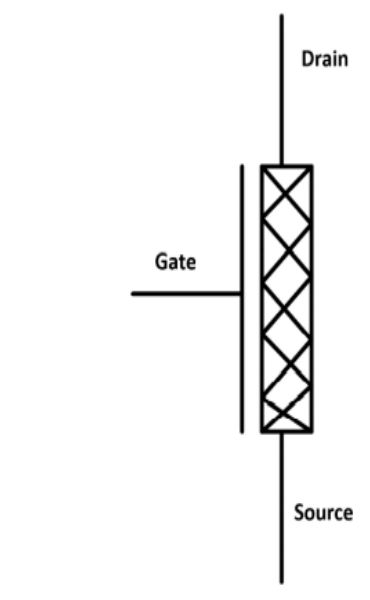

Fig. 2: Symbol of the model

are distributed evenly across the surface of nanotube. The Poisson equation in Eq. 9] is self-consistently solved in Eq. (4) with the Schrodinger equation.

For circuit simulations, a symbol was developed. Fig. 2 shows the symbol of SB-

\section{TFET.}

\section{ANT LION OPTIMIZATION}

Ant lion optimization (ALO) is an advanced computer method, guided by the unusual hunting behaviour of antlions in the hunting of their favourite prey, i.e., ants. In nature, each ant moves randomly during the hunt for prey. To prevent the ants from overshooting, random ant walks need to be normalized as follows.

$$
X_{j}^{t}=\frac{\left(X_{j}^{t}-\min _{j}\right) \times\left(d_{j}^{t}-c_{j}^{t}\right)}{\max _{j}-\min _{j}}+c_{j}^{t}
$$

where $\min _{j}$ and $\max _{j}$ are the minimum and maximum random walk in the $j^{\text {th }}$ variable, $c j^{t}$ and $d j^{t}$ represent the minimum and maximum of $j^{t h}$ variable in the $t^{t h}$ iteration. By adding the minimum and maximum of a variable to the location of the antlion, the ALO algorithm determines the new minimum and maximum of each variable in each iteration. Therefore, the ants' random walks are influenced as follows: 


$$
c_{j}^{t}=A L_{i}^{t}+c^{t}
$$

$$
d_{j}^{t}=A L_{i}^{t}+d^{t}
$$

For $t^{t h}$ iteration, $c^{t}$ and $d^{t}$ are the minimum and maximum of all variables respectively and $i^{t h}$ antlion at $t^{t h}$ iteration position is shown by $A L_{i}^{t}$. After that, the adaptive decrement of minimum and maximum values of variable are in following: $c^{t}=\frac{c^{t}}{R}, d^{t}=\frac{d^{t}}{R}$, and hence, the range variable moves toward the position of the antlion.

The ratio $R=10^{w} \frac{t}{T}$, where $t$ is the current iteration and $T$ is the maximum number of iterations, and $w$ is a constant, helps to control the precision level of exploitation. It is captured by an antlion as the ant hits the bottom of the pit. In addition, an antlion updates its location to increase its chances of attracting new ants in the following ways:

$$
A L_{i}^{t}=A n t_{j}^{t} \text { iff }\left(A n t_{j}^{t}\right)<f\left(A L_{i}^{t}\right)
$$

Where $t$ displays the current iteration, and Ant $j^{t}$ displays $j^{t h}$ ant at the location of $t^{t h}$ iteration. The fittest antlion is considered the elite, and during iterations it influences the motion of all the ants. The location of an ant, therefore, is as follows:

$$
A n t_{j}^{t}=\frac{R_{A}^{t}+R_{E}^{t}}{2}
$$

where $R_{A}^{t}$ is the random walk of the ant around a specific antlion, and $R_{E}^{t}$ is the random walk of the ant around the elite antlion.

For the distribution of the solution in the archive, ALO uses a niche approach, where the neighbourhood of each solution is explored in a predefined radius. From the solutions which have the least populated area, the ALO algorithm selects antlions. The likelihood that a solution set from the archive is selected is: $P_{j}=\frac{k}{N_{j}}$, where $k>1$ and $N_{j}$ are the number of solutions in the $j^{t h}$ neighbourhood. When the archive is wholly occupied, new solutions are replaced with the solutions that have the most populated neighbourhood. The probability of a solution being omitted is: $P j=\frac{k}{N j}$. The roulette wheel is used by ALO and Eq. 11] to select a non-dominated solution from the archive.

In terms of discovery and exploitation, ALO gives superior results [21]. Good exploration means that the possible areas of the search space are adequately investigated and prevents the local optima from trapping the algorithm, which is ensured by 
the random walks of ants near the antlion and the random selection of antlions. The shrinking boundaries of the pit, on the other hand, ensure successful utilisation.

\subsection{General steps of ALO}

The general steps of ALO (as shown in Fig. 33 to change these two sets and eventually estimate the global optimum for a given optimization the problem is as follows.

1. The ant set is initialized with random values and are the main search agents in the ALO.

2. The fitness value of each ant is evaluated using an objective function in each iteration.

3. Ants move over the search space using random walks around the antlions.

4. The population of antlions is never evaluated. In fact, antlions assumed to be on the location of ants in the first iteration and relocate to the new positions of ants in the rest of iterations if the ants become better.

5. There is one antlion assigned to each ant and updates its position if the ant becomes fitter.

6. There is also an elite antlion which impacts the movement of ants, regardless of their distance.

7. If any antlion becomes better than the elite, it will be replaced with the elite.

8. Steps (ii) to (vii) are repeatedly executed until the satisfaction of an end criterion.

9. The position and fitness value of the elite antlion is returned as the best estimation for the global optimum.

\subsection{Problem objectives}

In the problem of optimization, we need to choose the different objective functions which will help in selecting the value of the thickness of $\mathrm{ZrO} O_{2}$. The purpose of this is to reduce the ambipolar current. Mathematically, it can be formulated as follows.

1. For what of value of $t_{o x}, \frac{I_{o n}}{I_{o f f}}$ would be optimum? In Eq. (3), the value of $I_{o n}$ and $I_{\text {off }}$ can be acquired.

2. The objective function, which is to be maximized, is the selection of material which has different values of permittivity. 


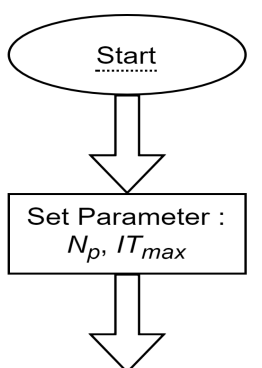

$$
\begin{gathered}
\text { - Randomly Initialize Populations, } A L_{s} \\
\text { - Evaluate quality of all solutions } \\
\text { - Select the best antlion } A L_{\text {best }}
\end{gathered}
$$

- Set current iteration to $1, I T=1$

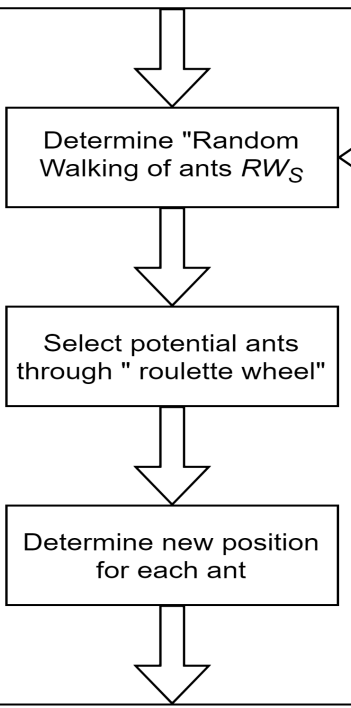

- Check lower and upper bounds for ants all variables - Evaluate quality of all solutions

Compare $A L_{s}$ and $A n t_{s}\left(s=1, \ldots, N_{p}\right)$ to keep better ones

- Name kept once to $A L s$

- Set $A L_{s}$ with the best fitness function to $A L_{\text {best }}$

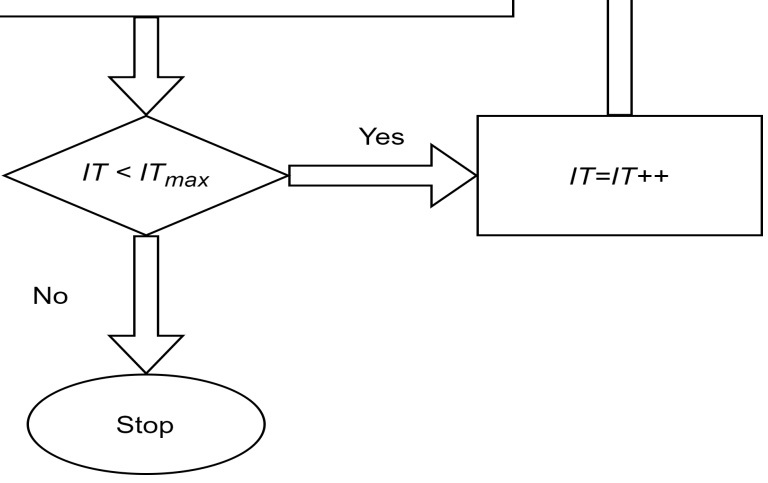

Fig. 3: Flow Chart of the Antlion Optimization 
Table 1: ALO parameters

\begin{tabular}{|l|l|}
\hline \multicolumn{2}{|c|}{-ALO Parameters- } \\
\hline Maximum iteration & 100 \\
ArchiveMaxSize & 100 \\
$\mathrm{~N}$ & 100 \\
Elite Position & zeros \\
\hline
\end{tabular}

For all these approaches, the main inspiration derives from biological processes in nature. Some of the standard features of all these unorthodox algorithms are that all of them are population-based, derivative-independent, and have a probabilistic approach. These algorithms begin with a set of trial solution, solution (called a parent population) and produce a fresh collection of solutions by carrying out proper transformations, which are unique to each algorithm, in a test solution in a stochastic manner. The best results can be obtained using heuristic optimization techniques.

For the parameters required for the CNTFET model, different optimization techniques are available in the nanoscale area to optimize the units. The high OFFstate leakage current under high reverse gate voltages due to band-to-band tunnelling (BTBT) is still a significant problem in CNTFETs. By varying the doping profile and asymmetry between source/drain regions, several researchers have tried to suppress the leakage current caused by BTBT.

In this work, we propose a technique of optimization based on ALO to suppress ambipolar behaviour. The main objective of the optimization process is to obtain the optimum value of the gate oxide's thickness and dielectric constant to achieve ambipolar conduction suppression without any loss of on-current.

The expected value of IDS,MOALO is the measured drain current based on the ALO computation. The numerical function targeted is given as IDS, NUM. Table 1 indicates the ALO parameters. Table 2 displays optimised values of the dielectric constant of the thickness and gate for good Ion / Ioff ratios. $49.91 \mathrm{~mm}$ is found to be the thickness, and 25 is the dielectric constant. Zirconium dioxide $\left(\mathrm{ZrO}_{2}\right)$ is, therefore, selected whose dielectric constant is near to 25. The SB CNTFET with 49.91 $\mathrm{mm} \mathrm{ZrO}_{2}$ dielectric was simulated with the optimized values extracted from ALO and is presented in the next section. 


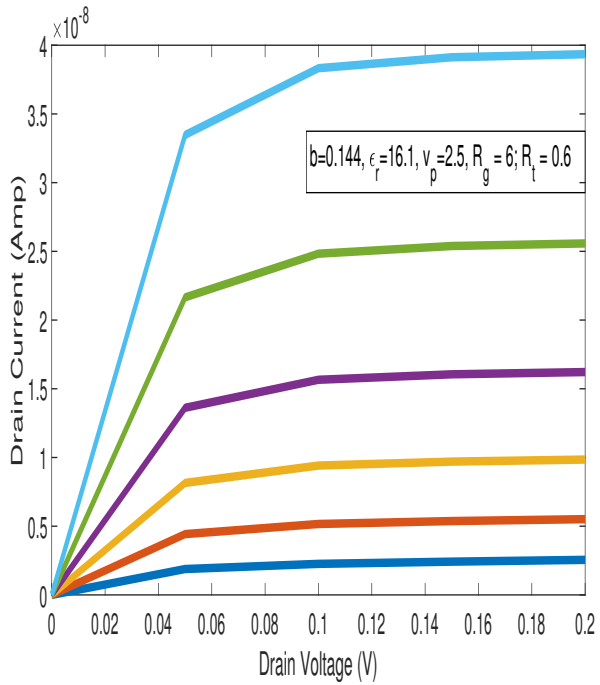

(a)

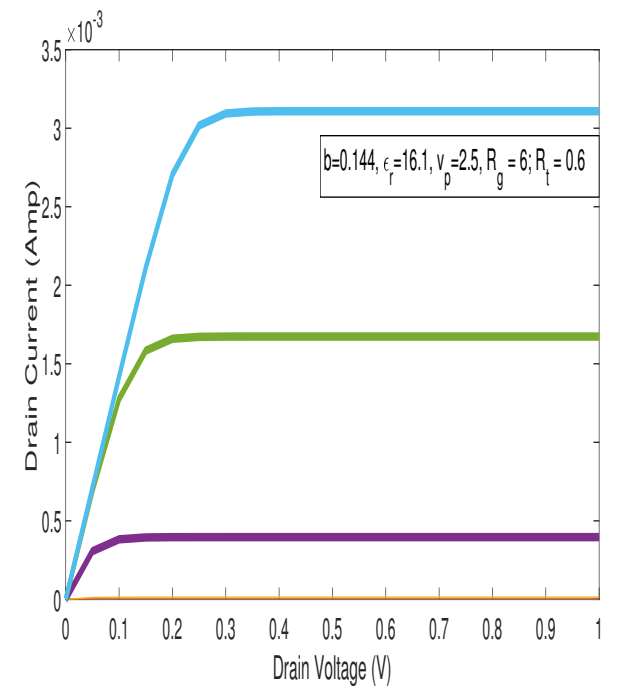

(c)

Fig. 4: Behaviour of Carbon Nanotube Transistor. (a) Drain current against Drain voltage, (b) Drain current against Gate voltage, (c) Drain current against Drain voltage, (d) Drain current against Gate voltage. The (a) and (b) characteristics curves have been plotted for thinkness of Gate oxide $\approx 49.91 \mathrm{~nm}$. However, The (c) and (d) characteristics curves have been plotted for thinkness $\approx 50.00 \mathrm{~nm}$. 


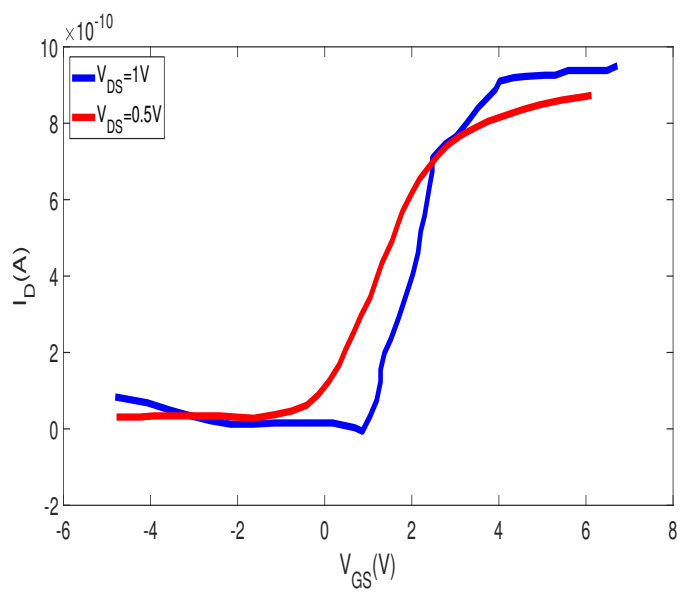

Fig. 5: Characteristic of device with thick gate oxide.

Table 2: Comparative Analysis of Optimized Parameters

\begin{tabular}{|l|l|l|}
\hline Quantity & Optimized values (Proposed Method) & Optimized values (PSO) [24] \\
\hline Gate oxide thickness, $t_{o x}$ & $49.91 \mathrm{~nm}$ & $50 \mathrm{~nm}$ \\
Gate dielectric constant, $\varepsilon$ & $25.00\left(\mathrm{ZrO}_{2}\right)$ & $25\left(\mathrm{ZrO}_{2}\right)$ \\
Off current, $\mathrm{I}_{\text {off }}$ & $10^{-19} \mathrm{~A}$ & $10^{-18} \mathrm{~A}$ \\
On current, $\mathrm{I}_{o n}$ & $10^{-6} \mathrm{~A}$ & $10^{-6} \mathrm{~A}$ \\
\hline
\end{tabular}

\section{Results and discussion}

Ideally, only by choosing a suitable metal with a high working function for holes and a low working function for electrons can be used to tune the SB height to some extent. It is impossible to adjust the SB height to zero because of the Fermi level pinning of the metal interfaces. However, Fermi level pinning for 1D channel materials is not known in the Metal Induced Gap States. As the SB heights can be tuned by the functional role of the metal contacts, this makes the metal contacted CNTFETs extremely desirable. Guo et al. conducted a comparative computational analysis of the impact of oxide thickness on CNTs' ambipolar behaviour [12,13]. They have numerically shown that the ambipolar conduct of CNTs is suppressed by a thick dielectric. The same outcome has been experimentally demonstrated. 


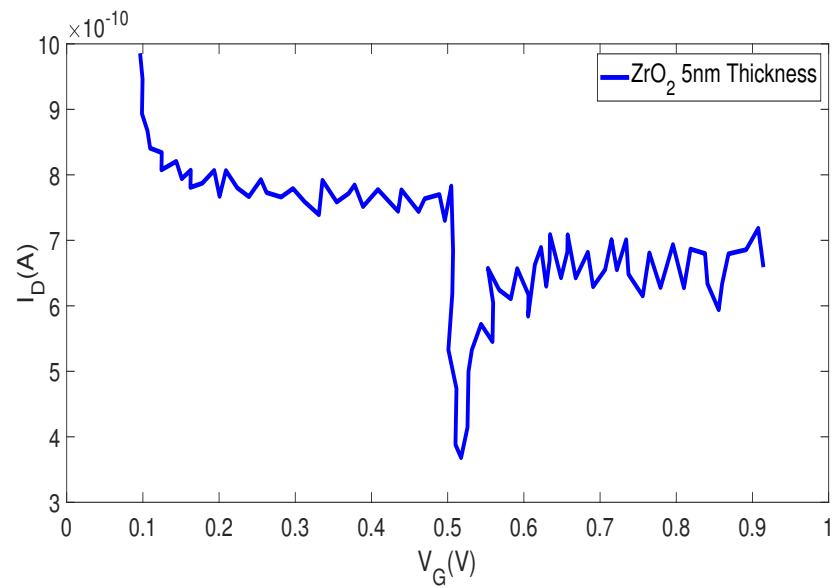

(a)

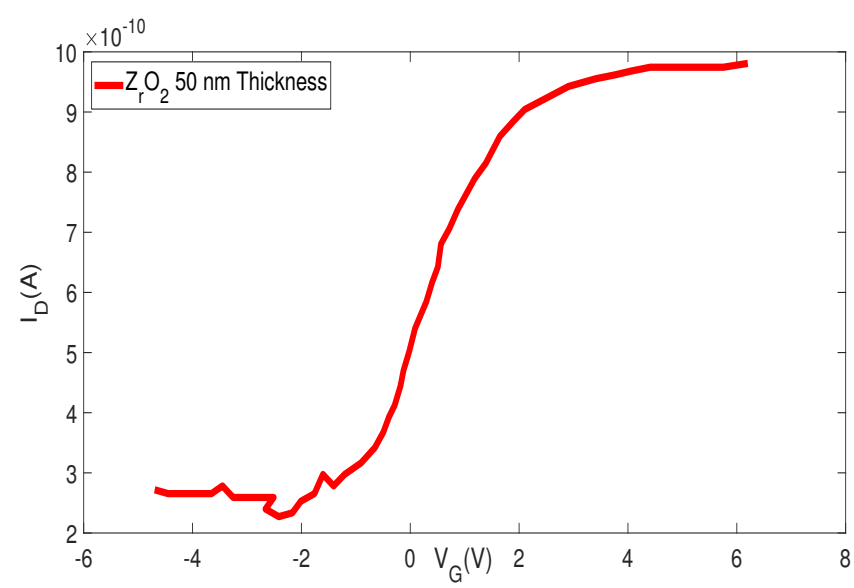

(b)

Fig. 6: $I_{D}$ versus $V_{G}$ for different thickness of $\mathrm{ZrO}_{2}$

\subsection{Suppression of Ambipolar Conduction in Schottky Barrier}

Aggressive scaling down of the thickness of the gate oxide makes the Schottky barriers very thin. This is due to the one-dimensional existence of CNTs. The drain current for different drain and gate voltages for distinct thickness levels have been shown in Fig. 4. Therefore, the tunnelling of electrons and holes takes place when the dielectric gate is thin, as both SBs (source and drain side) become transparent, resulting in ambipolar conduction. The gate oxide thickness determines the length scale over 

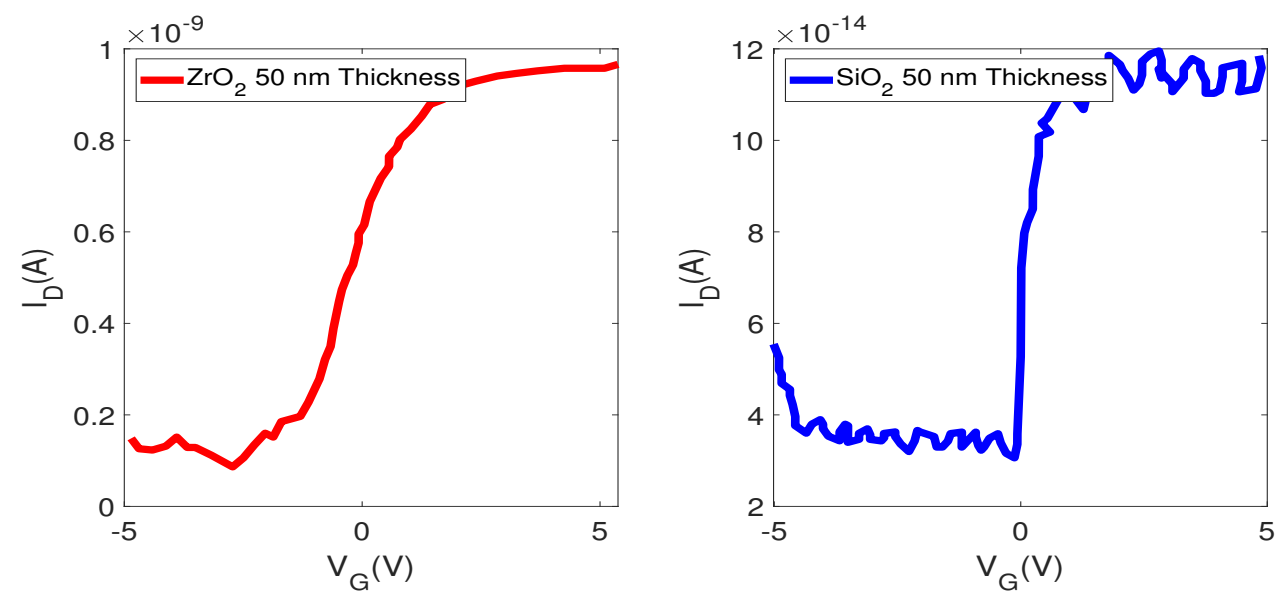

Fig. 7: Comparison graph with different dielectric.

which the bands bend at the source/drain metal contacts. The SBs are not transparent for tunnelling when the gate dielectric is dense. The gate to the source voltage adjusts the band such that electrons or holes tunnelling takes place. As shown in Fig. 6(a), the $I_{D}-V_{G}$ characteristics of the ambipolar devices present a V-shaped structure. In devices with thick dielectric gates, due to the trapped charges in the oxide, the minimum of the V-shaped plot is moved. It is possible to observe the suppression of ambipolar behaviour. The on-current due to tunnelling is, however, decreased due to the increased thickness of the gate oxide layer. Dielectric such as $\mathrm{ZrO}_{2}$ is used to improve the on-current due to thermionic emission. The on-current is, therefore, not affected. A. ray has shown that through tube diameter modulation with thin dielectrics, on-current (Ion) can be improved [30]. The Ion / Ioff ratio is demonstrated to be stronger for smaller diameters $(0.6 \mathrm{~nm})$ of the nanotube, but the on-current is weak. The result was higher on-current with nanotubes with a diameter of $2 \mathrm{~nm}$, but the Ion / Ioff ratio is low. By the thickness of the gate oxide to inhibit ambipolar activity without affecting the on- current, the proposed work modulates the thickness of the barrier. An experiment was performed to measure the $I-V$ characteristics of a system with a gate oxide like $\mathrm{SiO}_{2}$ with a thickness of $49.91 \mathrm{~mm}$. Suppression of the ambipolar conduction reveals the features, but the on-current was less (in the order of pico Amps). However, for a system with gate oxide like $\mathrm{ZrO}_{2}$ with the same thickness ( $49.91 \mathrm{~mm}$ ), the measured characteristics showed a higher current while suppressing 
the ambipolar conduction. The current is in the order of $\mu \mathrm{A}$. It is demonstrated in Fig. 5. The suppression is due to the thickness of gate, while the greater on-current is due to the oxide of the gate. It is thus validated that the increased gate oxide thickness suppresses the ambipolar activity and provides strong on-current. A comparative graph showing the on-current reduction for a gate oxide and the on-current increase for a gate oxide is shown in Fig. 6. In Fig. 7, we have shown the performance of $I_{D}$ for distinct values of $V_{G}$ under dielectrics of different oxides. In $\mathrm{SiO}_{2}$, the fluctuation in the current is predominant. Therefore, $\mathrm{ZrO}_{2}$ is more acceptable than $\mathrm{SiO}_{2}$. The $I_{D}-V_{G}$ characteristic for the device with thin gate oxide is shown in Fig. 6.a). The SB CNTFET with thin gate oxide exhibited ambipolar behaviour. The reason behind this is that the SB is thin and transparent to electrons and holes when the gate oxide is thin. Fig. 6.b) shows the actual behaviour of thin SB CNTFETs where the conduction is ambipolar due to the increased band to band tunnelling of holes. It happens because of the barrier lowering at the drain side for drain voltages greater than the gate voltage. The thickness of the barrier at the drain side is also reduced, thereby contributing tunnelling current. This causes a damaging effect on the device operation in both the on and off state.

\section{Conclusion}

The $I_{D}-V_{G}$ characteristics of the device with thick gate oxide, $t_{o x}$ of $49.91 \mathrm{~mm}$ for $V_{D}=0.5 \mathrm{~V}$ and $1 \mathrm{~V}$, did not exhibit ambipolar behaviour. Such devices exhibit unipolar characteristics as compared to that of similar devices fabricated with thin gate oxide. Thick SB is the reason for the suppression of the ambipolar conduction, which is the result of the thicker gate oxide. The SB limits the current at the metal-CNT contact. By changing the thickness of the tunnelling barrier, the fringing field of the gate modulates the current. It is concluded that, in the proposed device, the conduction of electrons for negative gate voltages is suppressed due to the asymmetry introduced by the thick gate oxide. However, the on-current is compensated by the dielectric.

\section{Ethics approval and consent to participate}

It is not applicable for this manuscript. 
7 Consent for publication

It is not applicable for this manuscript.

\section{Availability of data and materials}

It is not applicable for this manuscript.

\section{Competing interests}

The authors declare that they have no competing interests.

10 Funding

The authors did not receive support from any organization for the submitted work.

290

It is not applicable for this manuscript.

\section{Compliance with Ethical Standards}

296 I confirm for the following

297 1. No financial support from any institution or authors.

298 2. No conflicts of interest.

299 3. No research involving animals.

4. No research involving humans as subjects. 
13.1 Disclosure of potential conflicts of interest

It is not applicable for this manuscript.

\subsection{Research involving Human Participants and/or Animals}

It is not applicable for this manuscript.

\subsection{Informed consent}

It is not applicable for this manuscript.

\section{References}

1. Abbas, I., Saeed, T., Alhothuali, M.: Hyperbolic two-temperature photo-thermal interaction in a semiconductor medium with a cylindrical cavity. Silicon 13(6), 1871-1878 (2021)

2. Arefinia, Z., Orouji, A.A.: Novel attributes in the performance and scaling effects of carbon nanotube field-effect transistors with halo doping. Superlattices and Microstructures 45(6), 535-546 (2009)

3. Atzori, L., Iera, A., Morabito, G.: The internet of things: A survey. Computer networks 54(15), 27872805 (2010)

4. Avouris, P., Appenzeller, J., Martel, R., Wind, S.J.: Carbon nanotube electronics. Proceedings of the IEEE 91(11), 1772-1784 (2003)

5. Bhatt, V.D., Joshi, S., Becherer, M., Lugli, P.: Flexible, low-cost sensor based on electrolyte gated carbon nanotube field effect transistor for organo-phosphate detection. Sensors 17(5), 1147 (2017)

6. Chen, W., Zhang, Z., Feng, Z., Chen, Y., Jiang, K., Fan, S., Iskander, M.: Measurement of polarized nano-material (pnm) for microwave applications. In: 2008 IEEE MTT-S International Microwave Symposium Digest, pp. 1577-1580. IEEE (2008)

7. Choi, J., An, S.J.: Backside metallization of ag-sn-ag multilayer thin films and die attach for semiconductor applications. Journal of Electronic Materials pp. 1-7 (2020)

8. Dürkop, T., Getty, S., Cobas, E., Fuhrer, M.: Extraordinary mobility in semiconducting carbon nanotubes. Nano letters 4(1), 35-39 (2004)

9. Eberhart, R., Kennedy, J.: A new optimizer using particle swarm theory. In: MHS'95. Proceedings of the Sixth International Symposium on Micro Machine and Human Science, pp. 39-43. Ieee (1995)

10. Elhadidy, H., Mahi, F., Franc, J., Musiienko, A., Dedic, V., Schneeweiss, O.: Calculations of highfrequency noise spectral density of different cdte metal-semiconductor-metal schottky contacts. Journal of Electronic Materials 48(12), 7806-7812 (2019)

11. Endoh, T., Momma, Y.: Study of 30-nm double-gate mosfet with halo implantation technology using a two-dimensional device simulator. IEICE transactions on electronics 90(5), 1000-1005 (2007) 
12. Guo, G., Mandal, M., Jing, Y.: A robust detector of known signal in non-gaussian noise using threshold systems. Signal Processing 92(11), 2676-2688 (2012)

13. Guo, J., Datta, S., Lundstrom, M.: A numerical study of scaling issues for schottky-barrier carbon nanotube transistors. IEEE transactions on electron devices 51(2), 172-177 (2004)

14. Huo, X., Zhang, M., Chan, P.C., Liang, Q., Tang, Z.: High frequency s parameters characterization of back-gate carbon nanotube field-effect transistors. In: IEDM Technical Digest. IEEE International Electron Devices Meeting, 2004., pp. 691-694. IEEE (2004)

15. Hwang, H., Lee, D.H., Hwang, J.M.: Degradation of mosfets drive current due to halo ion implantation. In: International Electron Devices Meeting. Technical Digest, pp. 567-570. IEEE (1996)

16. Ieong, M., Narayanan, V., Singh, D., Topol, A., Chan, V., Ren, Z.: Transistor scaling with novel materials. Materials today 9(6), 26-31 (2006)

17. Javey, A., Guo, J., Wang, Q., Lundstrom, M., Dai, H.: Ballistic carbon nanotube field-effect transistors. nature 424(6949), 654-657 (2003)

18. Javey, A., Kong, J.: Carbon nanotube electronics. Springer Science \& Business Media (2009)

19. Jiang, L., Li, T., Jing, N., Kim, N.S., Guo, M., Liang, X.: Cnfet-based high throughput simd architecture. IEEE Transactions on Computer-Aided Design of Integrated Circuits and Systems 37(7), 1331-1344 (2017)

20. John, D., Castro, L., Pereira, P., Pulfrey, D.: A schrödinger-poisson solver for modeling carbon nanotube fets. In: Proc. NSTI Nanotech, vol. 3, pp. 65-68 (2004)

21. Kilic, H., Yuzgec, U., Karakuzu, C.: A novel improved antlion optimizer algorithm and its comparative performance. Neural Computing and Applications 32(8), 3803-3824 (2020)

22. Lin, Y.M., Appenzeller, J., Avouris, P.: Ambipolar-to-unipolar conversion of carbon nanotube transistors by gate structure engineering. Nano Letters 4(5), 947-950 (2004)

23. Liu, F., Chen, W., Zhang, Z., Feng, Z., Chen, Y., Zhang, H.: Measurement on dipole antenna with light polarized nano-material (pnm) textile reflector. In: 2009 IEEE MTT-S International Microwave Symposium Digest, pp. 1069-1072. IEEE (2009)

24. Monica, P.R., Sreedevi, V.: Suppression of ambipolar conduction in schottky barrier carbon nanotube field effect transistors: Modeling, optimization using particle swarm intelligence, and fabrication. Computer Modeling in Engineering \& Sciences 119(3), 577-591 (2019)

25. Naderi, A., Keshavarzi, P., Orouji, A.A.: Ldc-cntfet: A carbon nanotube field effect transistor with linear doping profile channel. Superlattices and Microstructures 50(2), 145-156 (2011)

26. Nouri-Bayat, R., Kashani-Nia, A.R.: Designing a carbon nanotube field-effect transistor with high transition frequency for ultra-wideband application. Engineering 9(1), 22-35 (2017)

27. Odintsov, A.A.: Schottky barriers in carbon nanotube heterojunctions. Physical Review Letters 85(1), 150 (2000)

28. Pourfath, M.: Numerical study of quantum transport in carbon nanotube-based transistors. Ph.D. thesis (2007)

29. Radosavljević, M., Heinze, S., Tersoff, J., Avouris, P.: Drain voltage scaling in carbon nanotube transistors. Applied Physics Letters 83(12), 2435-2437 (2003)

30. Raychowdhury, A.: Designing low-power and high-performance digital circuits with carbon nanotube transistors. Ph.D. thesis, Purdue University (2007)

31. Soref, R.: Silicon photonics: a review of recent literature. Silicon 2(1), 1-6 (2010) 
32. Thai, T.T., Yang, L., DeJean, G.R., Tentzeris, M.M.: Nanotechnology enables wireless gas sensing. IEEE Microwave Magazine 12(4), 84-95 (2011)

33. Xuan, C.T., Thuy, N.T., Luyen, T.T., Huyen, T.T., Tuan, M.A.: Carbon nanotube field-effect transistor for dna sensing. Journal of electronic materials 46(6), 3507-3511 (2017)

34. Yan, X., Xiao, Y., Li, Z.: Effects of intertube coupling and tube chirality on thermal transport of carbon nanotubes. Journal of applied physics 99(12), 124305 (2006)

35. Yang, K., Yi, Z., Jing, Q., Yue, R., Jiang, W., Lin, D.: Sonication-assisted dispersion of carbon nanotubes in aqueous solutions of the anionic surfactant sdbs: The role of sonication energy. Chinese science bulletin 58(17), 2082-2090 (2013)

36. Yang, Q., Scott, D., Chung, T., Stillman, G.: Optimization of emitter cap growth conditions for ingap/gaas hbts with high current gain by lp-mocvd. Journal of Electronic Materials 29(1), 75-79 (2000)

37. Yoon, Y., Fodor, J., Guo, J.: A computational study of vertical partial-gate carbon-nanotube fets. IEEE transactions on electron devices 55(1), 283-288 (2007)

38. Zanchetta, S., Todon, A., Abramo, A., Selmi, L., Sangiorgi, E.: Analytical and numerical study of the impact of halos on short channel and hot carrier effects in scaled mosfets. Solid-State Electronics 46(3), 429-434 (2002) 


\section{Supplementary Files}

This is a list of supplementary files associated with this preprint. Click to download.

- article.bib

- paralist.sty

- spbasic.bst

- spmpsci.bst

- spphys.bst

- svglov3.clo

- svjour3.cls 